\title{
The modeling of atom - neutral collisions for beam emission spectroscopy applications ${ }^{\star}$
}

\author{
O. Asztalos ${ }^{1, a}$, B. Szondy ${ }^{1}$, K. Tökési ${ }^{2,3}$, and G.I. Pokol ${ }^{1,4}$ \\ 1 Institute of Nuclear Techniques, Budapest University of Technology and Economics, Muegyetem rkpt 3, \\ 1111 Budapest, Hungary \\ 2 Institute for Nuclear Research, Hungarian Academy of Sciences (Atomki), 4001 Debrecen, Hungary \\ 3 ELI-ALPS, ELI-HU Non-profit Ltd., Dugonics ter 13, 6720 Szeged, Hungary \\ 4 Wigner RCP, Hungarian Academy of Science, 1121 Konkoly-Thege Miklos 29-33, Budapest, Hungary
}

Received 18 December 2018 / Received in final form 11 March 2019

Published online 1 June 2019

(C) The Author(s) 2019. This article is published with open access at Springerlink.com

\begin{abstract}
The collisional radiative models used in the modeling of beam emission spectroscopy diagnostics neglect atom-atom collisions because of a lack of sufficiently detailed atomic data. Filling this scantiness we performed a classical trajectory Monte Carlo simulations to calculate the cross sections for various channels in collisions between $\mathrm{H}+\mathrm{H}_{2}$ and $\mathrm{Li}+\mathrm{H}_{2}$ for a wide range of projectile energies. Based on the calculated cross sections, a simplified version of the collisional radiative model has been derived. We show that the model is suitable to obtain the beam attenuation in neutral gases outside of the confined plasma region. A strong density dependence has been found for each beam species.
\end{abstract}

\section{Introduction}

Beam emission spectroscopy (BES) is an active plasma diagnostic used for density measurements, which has sufficient spatial and temporal resolutions for the study of turbulent density fluctuations and associated flows [1]. A high energy neutral beam of $20-100 \mathrm{keV}$ is shot into the plasma, consisting of hydrogen isotopes or light alkali metal isotopes with only one valence electron. While attenuating, the beam atoms can be excited into higher states through various collisional processes with plasma particles (electrons, ions, impurities and neutrals) and spontaneously emit photons, which can be detected by an independent observation system. Plasma density [2] and fluctuations [3] measurement are based on the detected light signal and its fluctuations, respectively. Forward modeling of BES diagnostics is integral to the plasma density reconstruction [4] and spatial localization of density fluctuation [5], which strongly depend on the range and accuracy of underlying collisional radiative models (CRMs). Forward modeling BES codes, e.g., RENATE [6], are equipped with a CRM for alkali atom and hydrogenic beam emission modeling which accounts for collisional excitation, de-excitation, charge exchange, ionization and spontaneous emission. Existing CRMs do not include beam atom interaction with neutral particles, due to a general lack of cross-sections

* Contribution to the Topical Issue "Many Particle Spectroscopy of Atoms, Molecules, Clusters and Surfaces (2018)", edited by Károly Tőkési, Béla Paripás, Gábor Pszota, and Andrey V. Solov'yov.

${ }^{\text {a }}$ e-mail: asztalos@reak.bme.hu handling collisional interactions of higher atomic states at BES relevant impact energies. Recent trends in fusion research show a renewed interest in the contribution of neutral particles located outside of magnetic confinement region, enforcing the need to extend current CRMs. Applications include:

- improved estimation of beam attenuation, relevant for diagniostics and high powered heating beams [7],

- validation of BES relative calibration procedures also shown in Fisher et al. [8],

- improvement of synthetic BES diganostics [9] aiding the study on the effect of neutrals on the dynamics of scrape-off layer (SOL) turbulence [10].

A classical trajectory Monte Carlo (CTMC) method [11] has been used to reproduce the existing measured neutral with neutral ionization cross-sections found in literature to establish the method as a suitable tool for the computation of new, not yet measured, beam atom impact with neutral cross-sections.

Collisional radiative models used in the modeling of beam emission spectroscopy diagnostics neglect the atomatom collisions due to a lack of sufficiently detailed atomic data. In this work, filling this scantiness we present a classical trajectory Monte Carlo method to calculate the cross sections for various channels in collisions between $\mathrm{H}+\mathrm{H}_{2}$ and $\mathrm{Li}+\mathrm{H}_{2}$ in a wide range of projectile energies. Based on the calculated cross sections, a simplified version of the collisional radiative model has been derived. We show that the model is suitable to obtain the beam attenuation in neutral gases outside of the confined plasma region. 


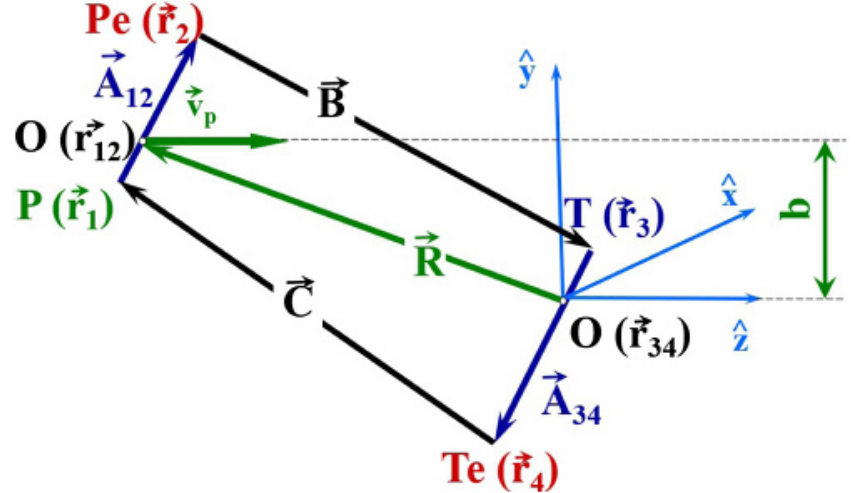

Fig. 1. The relative vectors involved in a 4 body interactions.

We organize the paper as follows: Section 2 introduces a CTMC method, ionization cross-section computations and comparisons are made with experimental measurements. Section 3 describes a CRM for neutral particle beam interaction. Beam attenuation is calculated using computed ionization cross-sections. It is shown that this model is suitable to improve the beam attenuation calculations in fusion experiments. Section 4 concludes on the viability of the CTMC method for cross-section generation as well as the inclusion of neutral particle with beam atom collisions into existing CRMs.

\section{Beam-neutral cross-sections}

Extensive work has been perfomed in the procurement of cross-sections for atomic rate calculations used by BES modeling, such as, Wutte et al. [12] which features an extended cross-section table with $n l$ resolved collisional cross-sections for lithium impact excitation, ionisation and charge exchange with electrons, protons and impurities for atomic levels up to $4 f$. This work also includes $L i+$ $\mathrm{H}_{2}$ ground state ionization cross-sections of the valence electron and collisional excitation to $2 p$. Similar work was done for sodium projectiles [13]. Earlier hydrogen impact cross-section work is more extensive, with regard to high energy $\mathrm{H}+\mathrm{H}_{2}$ collisions [14-16] but this lacks the detailed cross-section tables as featured for electron and proton impact interactions needed for beam evolution calculations.

\subsection{Classical trajectory Monte Carlo}

The classical trajectory Monte Carlo (CTMC) is a nonperturbative method that classically deals with collisions. CTMC has been shown to be effective in the calculation of ionization and capture cross-sections for ion atom collisions $[21,22]$.

In this work, a four body approximation is applied for the modeling of collisions between $L i$ and $H$ projectiles with $\mathrm{H}_{2}$ targets, (see Fig. 1). The interaction between the colliding particles is governed by the Coulomb force and described by a set of non-relativistic Newtonian equations:

$$
m_{i} \frac{d^{2} \mathbf{r}_{\mathbf{i}}}{d t^{2}}=\sum_{j=1, i \neq j}^{4} Z_{i} Z_{j} \frac{\left(\mathbf{r}_{\mathbf{i}}-\mathbf{r}_{\mathbf{j}}\right)}{\left|\mathbf{r}_{\mathbf{i}}-\mathbf{r}_{\mathbf{j}}\right|^{\mathbf{3}}}
$$

where $m_{i}, Z_{i}$ and $\mathbf{r}_{\mathbf{i}}$ are the mass, charge and relative position of the $i$ th particle, respectively. Figure 1 shows the schematic diagram of the calculated collision system. In our recent case both the target $\left(\mathrm{H}_{2}\right)$ and the projectiles $(L i, H)$ were considered as 2 bodies. In this simplest possible approach the $\mathrm{H}_{2}$ molecule is modeled by a hydrogentype atom with one active electron bound to the $\mathrm{H}_{2}^{+}$ion with an effective binding energy $\left(E_{\text {eff }}\right)$ and an effective core charge $\left(Z_{\text {eff }}\right)$. For $H_{2}$ target we used $Z_{\text {eff }}=1.165$ and $E_{\text {eff }}=0.567$ a.u. This approximation was successfully used in the investigation of the $\mathrm{H}_{2} \mathrm{O}$ molecule by several groups, leading to a good agreement between theoretical predictions and experimental data [17-20]. For the multilectron $\mathrm{Li}$ projectile, we take into the $2 s$ electron of the $\mathrm{Li}$ atom. The effective charge of 1.3 and binding energy of $0.198 \mathrm{a} . \mathrm{u}$. were used to model the $2 s$ orbital of the $\mathrm{Li}$ atom. Accordingly the $\mathrm{H}$ atom was mimicked with $Z=1$ and with $E=0.5$ a.u. $\mathbf{A}_{\mathbf{1 2}}, \mathbf{A}_{\mathbf{3 4}}$ constitute the positions of bound electrons to projectile and target, respectively; $\mathbf{R}$ the relative position between target and projectile centers of mass and $b$ the impact parameter. The collision is initialized with given projectile velocity $\left(\mathbf{v}_{\mathbf{p}}\right)$, at a suitable distance between projectile and target to ensure a negligible electrostatic interaction between the bound systems.

Nine different classical states were considered and the total projectile ionization cross-section was computed from the relevant exit channels that resulted in projectile electron loss. The final cross-sections for the exit channels are computed from a large number of test cases:

$$
\sigma=\frac{2 \pi b_{\max }}{T_{N}} \sum_{j=1}^{T_{N}^{i}} b_{j}^{(i)}
$$

where $T_{N}$ is the total number of trajectories computed with impact parameters less than $b_{\max }, T_{N}^{i}$ are the number of trajectories relevant for the investigated $i$ th channel with $b_{j}^{(i)}$ the corresponding impact parameters randomly chosen following a uniform distribution.

\subsection{Results}

The ionization cross-sections of projectile atoms in hydrogen gas were calculated. In these simulations, $2 \times 10^{6}$ primary trajectories were followed based on a four body model. For the total projectile ionization cross-section computations four relevant exit channels were considered:

$$
\begin{aligned}
& X+H_{2} \rightarrow X^{+}+H_{2}+e_{p} \\
& X+H_{2} \rightarrow X^{+}+H_{2}+e_{t} \\
& X+H_{2} \rightarrow X^{+}+H_{2}^{+}+2 e \\
& X+H_{2} \rightarrow X^{+}+H_{2}^{-} .
\end{aligned}
$$

Figure 2A shows the total projectile ionization crosssection (red) as well as all the contributing exit channels 


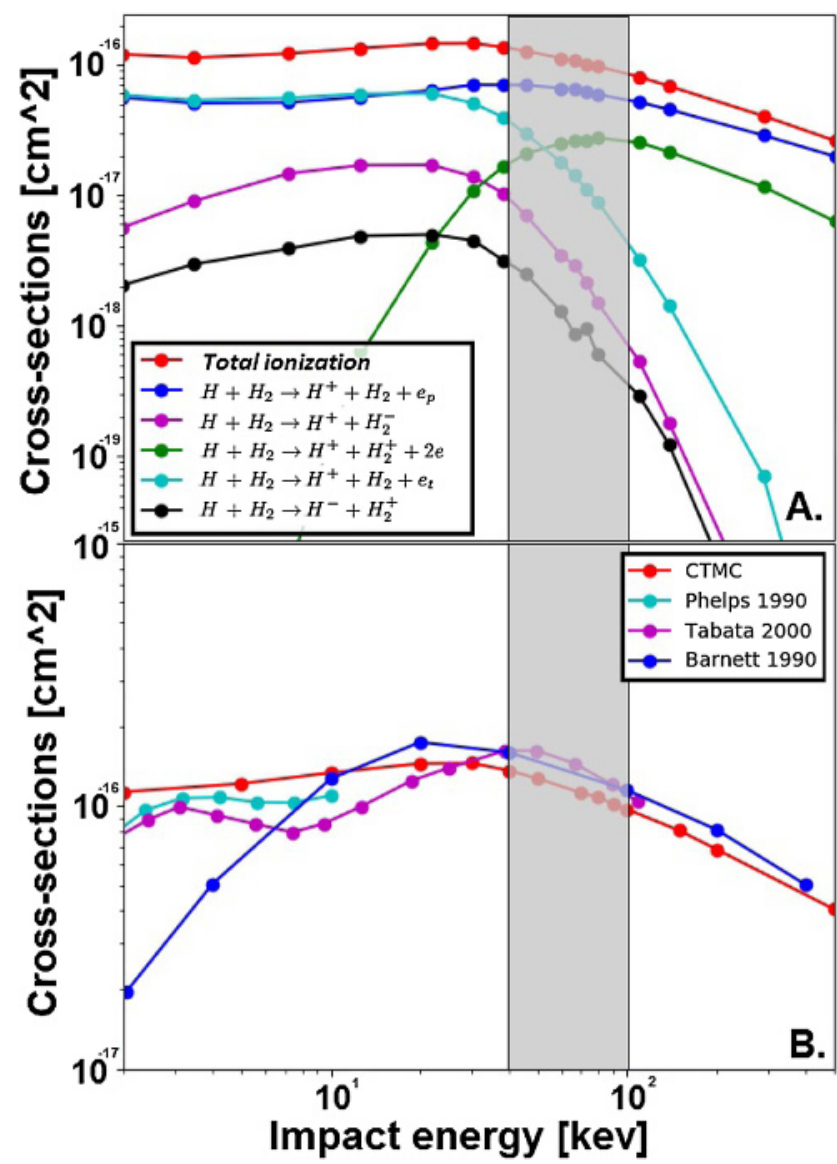

Fig. 2. A. Total ionization cross-section of a $\mathrm{H}(n=1)$ projectile at BES relevant impact energies on $\mathrm{H}_{2}$ molecules(red) with all the contributing ionization exit channels. B. Comparison of the total $\mathrm{H}+\mathrm{H}_{2}$ ionization cross-section calculated by the CTMC method (red) with previously measured data.

for $\mathrm{H}+\mathrm{H}_{2}$ interaction. The grey shaded area marks the cross-sections that are relevant for BES impact energies. The lower impact energy region is dominated by direct projectile ionization (blue) (Eq. (3)) as well as projectile ionization resulted from target ionization accompanied by projectile charge exchange (cyan) (Eq. (4)).

Both have similar values up to an impact energy of $20 \mathrm{keV}$ after which direct projectile ionization becomes the dominant mechanism. Target (purple) (Eq. (6)) electron capture mechanism has a similar decreasing trend as a function of impact energy. The projectile ionization by target electron capture is an obvious inclusion into the total projectile ionization cross-section. Simultaneous target and projectile ionization (green) (Eq. (5)) is most effective at impact energies in range of $100 \mathrm{keV}$, increasing with impact energy to one order of magnitude less than the dominant ionization mechanism. Similar features were observed for the ionization of lithium beam atoms through collisions with hydrogen molecules. The dominance of direct projectile ionization is more contested in the lower impact energy range. The relative contributions of other ionization mechanisms are stronger by a factor $2-5$ in case of $\mathrm{Li}+\mathrm{H}_{2}$ collisions with respect to the $\mathrm{H}+\mathrm{H}_{2}$ collisions. The total ionization cross-section is derived by $\sigma_{a}=\sum_{i} \sigma_{a i}$, where the $\sigma_{a i}$ characterize the individual exit channels. Both lithium and hydrogen ionization cross-sections have been compared to previously published experimental cross-section measurements. A good agreement was found for BES relevant beam energies, which are in a typical range of $40-100 \mathrm{keV}$ (Fig. 2B). The lower impact energy range is somewhat contestable due to differing trend and values with the cross-sections featured by Barnett et al. [23]. However, there is an overall good agreement with Phelps et al. [14] and Tabata et al. [24]. Similarly good agreement was achieved for lithium ionization cross-sections with hydrogen molecules with impact energies above $10 \mathrm{keV}$ with cross-section are featured in Wutte et al. [12].

$$
X+H_{2} \rightarrow X^{-}+H_{2}^{+} .
$$

Negative ion formation is considered a beam attenuation mechanism (see Eq. (7)). A change in projectile charge by projectile electron capture results in the projectile being lost from the beam by following magnetic field lines. Figure 2A shows the energy dependence of the projectile electron capture cross-section (black), having a similar trend as the target electron capture cross-section (purple) (Eq. (6)) and having values two orders of magnitude lower than the total ionization cross-section. The total electron loss cross-section that results in beam attenuation in high magnetic fields applied to the CRM is as follows: $\sigma_{a}^{\text {loss }}=\sigma_{a}^{\text {ion }}+\sigma_{a}^{(7)}$.

\section{Beam attenuation in neutral gas}

An adequate inclusion of beam atoms with neutral particle interactions (Eq. (8)) into existing CRMs, such as featured in RENATE, require the rate equation system to be extended by:

$$
\begin{aligned}
\frac{d n_{i}}{d t} & =\sum_{a} N_{a}\left(R_{a}^{s(i)}-R_{a}^{d(i)}\right)+S^{(i)} \\
R_{a}^{d(i)} & =n_{i}\left(\sum_{j=i+1}^{m} R_{i \rightarrow j}^{\mathrm{ex}}+\sum_{j=0}^{i-1} R_{i \rightarrow j}^{\mathrm{dex}}+R_{i \rightarrow+}^{\mathrm{loss}}\right) \\
R_{a}^{s(i)} & =\sum_{j=0}^{i-1} n_{j} R_{j \rightarrow i}^{\mathrm{ex}}+\sum_{j=i+1}^{m} n_{j} R_{j \rightarrow i}^{\mathrm{dex}} \\
S^{(i)} & =n_{j} \sum_{j=i+1}^{m} A_{j \rightarrow i}-n_{i} \sum_{j=0}^{i-1} A_{i \rightarrow j}
\end{aligned}
$$

where $n_{i}$ and $n_{j}$ are the electron population on the $i$ th and $j$ th levels, $N_{a}$ is the ath type of neutral particle density and the $R_{a}^{s(i)}, R_{a}^{d(i)}, S^{(i)}$ correspond to electron source, electron drain and spontaneous emission terms, respectively to and from the $i$ th level. Equation (9) gives the electron loss mechanisms from the ith level by means of collisional excitation to higher atomic states $\left(R_{i \rightarrow j}^{\mathrm{ex}}, i<j\right)$, collisional de-excitation to lower atomic 
states $\left(R_{i \rightarrow j}^{\text {dex }}, i>j\right)$ and electron loss form the atom $\left(R_{i \rightarrow+}^{\text {loss }}\right)$ described by the corresponding reaction rates. Equation (10) gives the electron gain mechanisms to the $i$ th level by means of collisional excitation from lower atomic states $\left(R_{j \rightarrow i}^{\mathrm{ex}}, i>j\right)$ and collisional de-excitation from higher atomic states $\left(R_{j \rightarrow i}^{\mathrm{dex}}, i<j\right)$. Electron gain by recombination is neglected. The above described terms effecting the electron population evolution are strongly dependent on the local density of the neutral particles along the beamline. Equation (11) gives the spontaneous emission terms to and from the $i$ th level.

A simplified CRM is put forward and studied in order to estimate the effects that neutral particle populations have on high energy atomic beams in conditions to be expected in fusion devices and further justify development of CRMs featuring atomic beam with neutral particle interactions. Excited states are neglected, resulting in the $-N_{a} n_{0} R^{\text {loss }}$ remaining term on the right hand side of in equation (8). "Turning off" higher atomic states, effectively neglecting multistage ionization and other electron loss mechanisms, results in a systematic underestimation of the beam attenuation but does not affect the order of magnitude. Most neutral particles effecting the beam evolution are located outside of the confined region of the plasma in the beam duct. The temperatures of the neutral particle populations in question are below the ionization threshold for atomic gases and below the molecular disassociation energy for molecular gases. A lithium or hydrogen atom accelerated to $50 \mathrm{keV}$ will observe the thermal gas below $1 \mathrm{eV}$ as stationary $(\mathbf{v} \rightarrow 0)$, resulting in following simplification of the rates:

$$
R=\langle\sigma v\rangle=\int\left[f_{m}(T, \mathbf{v})\left|\mathbf{v}-\mathbf{v}_{\mathbf{b}}\right| \sigma_{a}\left(\mathbf{v}-\mathbf{v}_{\mathbf{b}}\right)\right] d \mathbf{v} \approx \sigma \mathbf{v}_{\mathbf{b}}
$$

where $f_{m}(T, \mathbf{v})$ gives a Maxwellian distribution function, $\left|\mathbf{v}-\mathbf{v}_{\mathbf{b}}\right|$ is the impact velocity which in current approximation corresponds to the beam velocity and $\sigma_{a}$ the corresponding ionization cross-section for the collision of the ath neutral particle with beam atoms. The transformation of the equation from a temporal grid to a spatial grid results in the following simple form given for beam attenuation:

$$
\frac{d n_{0}}{d x}=-n_{0} \sum_{a} N_{a} \sigma_{a}^{\text {loss }}
$$

where $N_{a}$ is the gas density, $n_{0}$ is the electron population in ground level and $\sigma_{a}^{\text {loss }}$ is the total electron loss cross-section for the beam atom with neutral particle in question, as discussed at the end of Section 2.2.

The most significant impact on beam attenuation by beam atom interaction with neutral particle populations is expected to occur along the beam line starting from the neutralizer, through the beam duct, port plug, up to the plasma surface. The flight path of beam atoms from the beam duct to plasma surface varies strongly on the size of fusion devices, from $1.5 \mathrm{~m}$ for medium sized devices, like EAST [3] and this distance is considerably increased upon the inclusion of the flight distance of the outer beam duct. The total beam atom flight path to reach the ITER plasma for the diagnostic neutral beam (DNB)

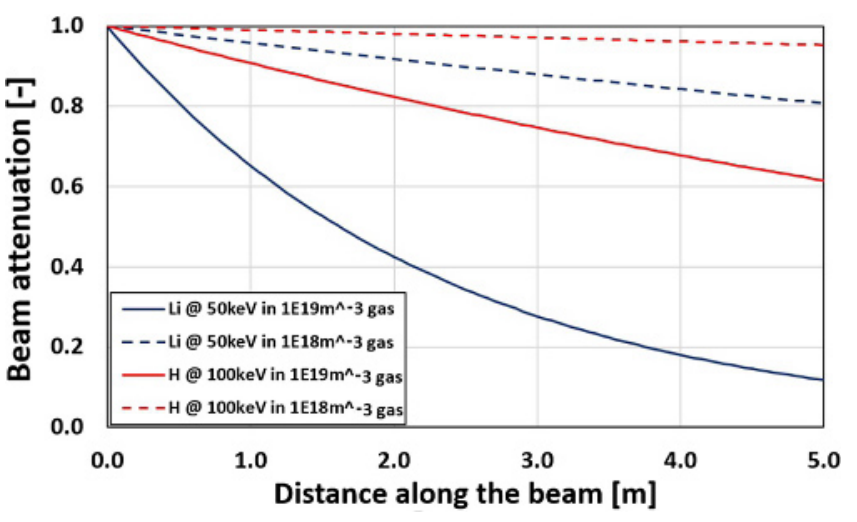

Fig. 3. Beam attenuation for hydrogen (red) and lithium (blue) beams in function of distance in $\mathrm{H}_{2}$ gasses of various density; solid for $10^{19} \mathrm{~m}^{-3}$ and dashed for $10^{18} \mathrm{~m}^{-3}$ gas densities.

is expected to be in the range of $20.7 \mathrm{~m}[25,26]$. There is no detailed information of neutral particle densities along the beam path therefore simple density profiles are assumed. Evolution of neutral particle populations is determined by particle sources such as the divertor, first wall and neutralizer; particle drains such as the vacuum system. The paper by Zhang et al. [27] discusses the effects of gas injection on the edge plasma and ion cyclotron resonance heating, which has the potential of causing neutral beams to operate within a higher density neutral particle population. Gas seeding [28] and wall coating [29] experiments have similar effects.

The aim of current contribution is to assess the density dependence of beam attenuation for various beam types in density ranges to be expected in the beam duct. A constant density along the beamline is assumed and this provides a fast assessment within an order of magnitude of expected beam attenuation along the flight path of the neutral beam. Figure 3 shows the expected beam attenuation for neutral densities of $10^{19} \mathrm{~m}^{-3}$ (solid lines) and $10^{18} \mathrm{~m}^{-3}$ (dashed lines) for lithium of $50 \mathrm{keV}$ (blue) and hydrogen $100 \mathrm{keV}$ (red). No relevant beam attenuation occurs for neutral particle densities in order of magnitude of $10^{17} \mathrm{~m}^{-3}$ or lower. For flight paths of neutral beams in medium sized machines, no significant impact is expected by neutral particle densities of $10^{18} \mathrm{~m}^{-3}$. Neutral particle densities of orders of magnitude $10^{19} \mathrm{~m}^{-3}$ or larger, can cause significant beam attenuation. Alkali diagnostic beams with a flight paths of $1-$ $3 \mathrm{~m}$ can loose up to $50 \%$ of their beam current. The beam attenuation for heating beams under similar circumstances features less dramatic effects. Beam attenuation in large machines, such as the ITER, can feature losses up to $20 \%$ for neutral densities between $10^{18} \mathrm{~m}^{-3}$ and $10^{19} \mathrm{~m}^{-3}$. These calculations are in agreement with the ITER DNB team $[25,26]$, where an experimental setup for the determination of realistic neutral particle density distribution in the ITER DNB beam duct was put forward.

Modeling beam attenuation in constant density neutral gases gives an approximation for the neutral particle densities at which beam atom interaction with said particles becomes significant. A strong density dependence was found, enforcing the need for better and more accurate 
neutral particle density models to be used in beam evolution calculations. Beam evolution calculations require development of a full CRMs to support such beam atom collisions with neutral particles.

\section{Conclusions}

A shortage of cross-sections for beam emission spectroscopy modeling has been identified, with regard to the interaction of beam atoms with neutral particles. Current literature holds measured cross-sections only for ground state valence electron ionization and excitation to higher atomic levels [14-16,23,24].

A 4-body classical trajectory Monte Carlo method was performed to calculate ground state ionization crosssections for hydrogen and lithium valence electron. All possible classical exit channels were identified and analyzed. It was found that the dominant ionization mechanism is direct projectile ionization, for both hydrogen and lithium beam species. Total ionization cross-sections have been compared to existing experimental measurements. Good agreement was found for impact energies relevant to BES diagnostics and thus the CTMC method was shown to be a viable for the calculation of further cross-sections missing from literature, such as collisional excitation or ionization from and to higher atomic levels. A collisional radiative model was derived for neutrals, based on existing CRMs. A simplified version of the CRM was used to determine the beam attenuation that could be expected in function hydrogen gas density. A number of hydrogen gas density scenarios were put forward and shown that plasma operations resulting in $H_{2}$ densities of larger than $10^{18} \mathrm{~m}^{-3}$ densities at the beam duct, may lead to significant beam losses. These results indicate the need for a full CRM model of beam atom interaction with neutral particles, which can lead to improvement of BES forward modeling. The improved CRM enables the modeling of the relative calibration processes which occur in neutral gasses, provides an initial condition for forward BES modeling and allows for the inclusion of neutrals in beam evolution process located in the SOL.

Open access funding provided by Budapest University of Technology and Economics (BME). The work was support by the National Research, Development and Innovation Office (NKFIH) Grant KH126886. Partial support by the ELI-ALPS project is also acknowledged. This work has been carried out within the framework of the EUROfusion Consortium and has received funding from the Euratom research and training program 2014-2018 under grant agreement No. 633053. The views and opinions expressed herein do not necessarily reflect those of the European Commission. We are thankful to Dr. Andrew Cheesman for the critical reading of the manuscript.

\section{Author contribution statement}

O. Asztalos: development of CRM, performance of beam attenuation calculations and CTMC calculations.
B. Szondy: development of CRM, performance of beam attenuation calculations and CTMC calculations. K. Tökesi: development of CTMC, performance of CTMC calculations. G.I. Pokol: development of CRM and performance of beam attenuation calculations.

Open Access This is an open access article distributed under the terms of the Creative Commons Attribution License (http://creativecommons.org/licenses/by/4.0/), which permits unrestricted use, distribution, and reproduction in any medium, provided the original work is properly cited.

\section{References}

1. F.M. Levinton, R.J. Fonck, G.M. Gammel, R. Kaita, H.W. Kugel, E.T. Powell, D.W. Roberts, Phys. Rev. Lett. 63, 2060 (1989)

2. J. Schweinzer, E. Wolfrum, F. Aumayr, M. Pockl, H. Winter, R.P. Schorn, E. Hinz, A. Unterreiter, Plasma Phys. Control. Fusion 34, 1173 (1992)

3. S. Zoletnik, G.H. Hu, B. Tal, D. Dunai, G. Anda, O. Asztalos, G.I. Pokol, S. Kalvin, J. Nemeth, T. Krizsanoczi, Rev. Sci. Instrum. 89, 063503 (2018)

4. D.I. Refy, M. Brix, R. Gomes, B. Tal, S. Zoletnik, D. Dunai, G. Kocsis, S. Kalvin, T. Szabolics, JET Contributors, Rev. Sci. Instrum. 89, 043509 (2018)

5. O. Asztalos, G.I. Pokol, S. Zoletnik, D. Guszejnov, G.H. Hu, R. Chen, G.S. Xu, in 44th European Physical Society Conference on Plasma Physics, EPS 2017 (2017) P4. 109

6. D. Guszejnov, G.I. Pokol, I. Pusztai, D. Refy, S. Zoletnik, M. Lampert, Y.U. Nam, Rev. Sci. Instrum. 83, 113501 (2012)

7. R.S. Hemsworth, D. Boilson, P. Blatchford, M. Dalla Palma, G. Chitarin, H.P.L. de Esch, F. Geli, M. Dremel, J. Graceffa, D. Marcuzzi, G. Serianni, D. Shah, M. Singh, M. Urbani, P. Zaccaria, New J. Phys. 19, 025005 (2017)

8. R. Fischer, E. Wolfrum, J. Schweinzer, ASDEX Upgrade Team, Plasma Phys. Control. Fusion 50, 085009 (2008)

9. A.H. Nielsen, V. Naulin, J. Juul Rasmussen, J. Olsen, A.S. Thryse, T. Eich, G.I. Pokol, O. Asztalos, N. Vianello, R. Coelho, D.I. Refy, B. Tal, G.H. Hu, N. Ning, EUROfusion MST1 Team, EUROfusion-IM Team, IAEA CN-258 (2018)

10. A.S. Thrysoe, L.E.H. Tophj, V. Naulin, J.J. Rasmussen, J. Madsen, A.H. Nielsen, Plasma Phys. Control. Fusion 58, 044010 (2016)

11. K. Tokesi, R.D. DuBois, T. Mukoyama, Eur. Phys. J. D 68, 255 (2014)

12. D. Wutte, R.K. Janev, F. Aumayr, M. Schneider, J. Schweinzer, J.J. Smith, H.P. Winter, At. Data Nucl. Data Tables 65, 155 (1997)

13. K. Igensberg, J. Schweinzer, I. Bray, D. Bridi, F. Aumayr, At. Data Nucl. Data Tables 94, 981 (2018)

14. A.V. Phelps, J. Phys. Chem. Ref. Data 19, 3 (1990)

15. I.D. Williams, J. Geddes, H.B. Gilbody, J. Phys. B: At., Mol. Phys. 15, 1377 (1982)

16. I.D. Williams, J. Geddes, H.B. Gilbody, J. Phys. B: At., Mol. Phys. 16, L765 (1983)

17. S. Otranto, R.E. Olson, Phys. Rev. A 77, 022709 (2008) 
18. L.F. Errea, C. Illescas, L. Mendez, B. Pons, I. Rabadan, A. Riera, Phys. Rev. A 76, 040701 (2007)

19. L. Mendez, L.F. Errea, C. Illescas, I. Rabadan, B. Pons, A. Riera, AIP Conf. Proc. 1080, 51 (2008)

20. S. Borbely, K. Tokesi, L. Nagy, Eur. Phys. J. D 59, 337 (2010)

21. R.E. Olson, A. Salop, Phys. Rev. A 16, 531 (1977)

22. K. Tokesi, G. Hock, Nucl. Instrum. Methods Phys. Res. B 86, 201 (1994)

23. C.F. Barnett, in Atomic Data for Fusion, ORNL-6086, (1990), Vol. I

24. T. Tabata, T. Shirai, At. Data Nucl. Data Tables 76, 1 (2000)

25. A. Chakraborty, C. Rotti, M. Bandyopadhyay, M.J. Singh, R.G. Nair, S. Shah, U.K. Baruah, R.S. Hemsworth, B. Schunke, IEEE Trans. Plasma Sci. 33, 248 (2010)
26. M.J. Singh, A.K. Chakraborty, M. Bandyopadhyay, J. Joshi, H. Patel, S. Shah, A. Gahlaut, A. Yadav, D. Parmar, D. Sharma, D. Singh, H. Tyagi, K. Joshi, K. Pandya, M.V. Nagaraju, M. Bhuyan, M. Patel, R. Yadav, S. Pillai, D. Boilson, J. Chareyre, B. Schunke, C. Rotti, 27th-IAEA Fusion Energy Conference, FIP/P1$40(2018)$

27. W. Zhang, P. Jacquet, E. Lerche, R. Bilato, V. Bobkov, D. Coster, Y. Feng, C. Guillemaut, M. Goniche, D. Harting, T. Lunt, J.-M. Noterdaeme, G. Szepesi, D. Van Eester and the JET Contributors, Nucl. Fusion 57, $056042(2017)$

28. A. Kallenbach for the ASDEX Upgrade Team and the EUROfusion MST1 Team, Nucl. Fusion 57, 102015 (2017)

29. Z. Sun, J.S. Hu, G.Z. Zuo, J. Ren, B. Cao, J.G. Li, D.K. Mansfield, the EAST team, Fusion Eng. Des. 89, 2886 (2014) 